\title{
The normal distribution
}

\author{
Douglas G Altman, J Martin Bland
}

$s$ is the 11th in a series of ccasional notes on medical statistics
FIG 1 (left)-Serum albumin values in 248 adults FIG 2 (right)-Normal distribution with the same mean and standard deviation as the serum albumin values

\section{Medical Statistics Laboratory, Imperial Cancer Research Fund, PO Box 123, London WC2A 3PX}

Douglas G Altman, Head

Department of Public Health Sciences, St George's Hospital Medical School, London SW170RE

$\mathrm{J}$ Martin Bland, reader in medical statistics

Correspondence to: Mr Altman.

BMF 1995;310:298
When we measure a quantity in a large number of individuals we call the pattern of values obtained a distribution. For example, figure 1 shows the distribution of serum albumin concentration in a sample of adults displayed as a histogram. This is an empirical
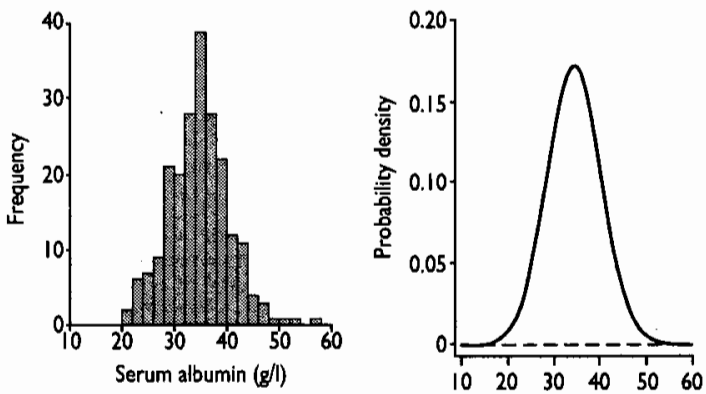

distribution. There are also theoretical distributions, of which the best known is the normal distribution (sometimes called the Gaussian distribution), which is shown in figure 2 . Although widely referred to in statistics, the normal distribution remains a mysterious concept to many. Here we try to explain what it is and why it is important.

In this context the name "normal" causes much confusion. In statistics it is just a name; statisticians often use a capital $\mathrm{N}$ to emphasise this and to clarify that Normality does not necessarily imply normality. Indeed, in some medical specialties normal distributions are rare.

Various methods of analysis make assumptions about normality, including correlation, regression, $t$ tests, and analysis of variance. It is not in fact necessary for the distribution of the observed data to be normal, but rather the sample values should be compatible with the population (which they represent) having a normal

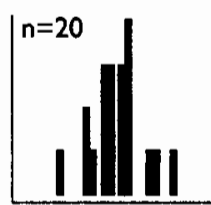

$n=50$
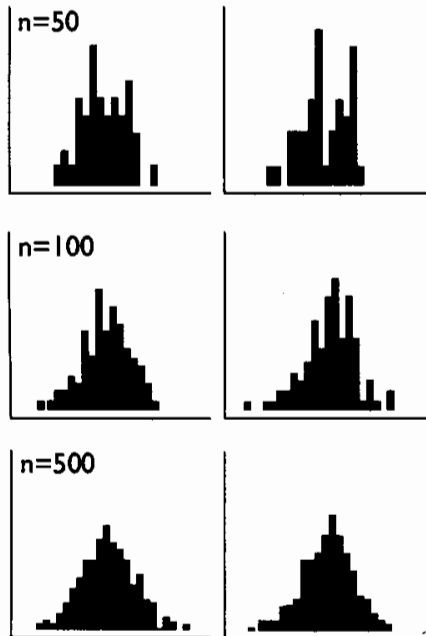

FIG 3-Random samples from normal distributions-five samples of size 20,50,100, and 500 distribution. Indeed, samples from a population is which the true distribution is normal will not neces? sarily look normal themselves, especially if the sample is small. Figure 3 shows the distributions of samples of different sizes drawn at random from normal distribu tions-few of the small samples look like a normal distribution, but the similarity increases as the sample्, size increases.

Although some statistical methods, such as the $t$ test are not sensitive to moderate departures fromi. normality, it is.generally preferable not to rely on this feature. Visual inspection of the distribution mag suggest whether the assumption of normality is reason able but, as figure 3 suggests, this approach is unio reliable. Significance tests and normal plots can be used to assess formally whether sample data are $\$$ plausible sample from a normal population.' When data do not have a normal distribution we can eitherr transform the data (for example, by taking logarithms or use a method that does not require the data to bs normally distributed. We consider these topics future notes.

The normal distribution has another essential place in statistics. Just as separate samples selected tל random from the same population will differ (fig 3) so will calculated statistics such as the mean bloo pressure. We can think of the means from manto samples as themselves also having a distribution. A ke theoretical result, called the central limit theorem underpins many methods of analysis. It states tha the means of random samples from any distribution will themselves have a normal distribution. As consequence, when we have samples of hundreds of observations we can often ignore the distribution of the data. Nevertheless, because most clinical studies are of a modest size, it is usually advisable to transform non' normal data, especially when they have a skewe distribution.

We can consider binary attributes in the same way? For example, the proportions of individuals witg asthma will vary from sample to sample. If having asthma is represented by the value 1 and not having asthma by the value 0 then the mean of these values the sample is the proportion of individuals with asthma. Thus a proportion is also a mean and wilp follow a normal distribution. These methods are not valid in small samples-some "exact" methods cax be used." Similar comments apply to some othes statistics, such as regression coefficients or standard $\mathbb{\Phi}_{\mathscr{\Phi}}$ ised mortality ratios, but for mortality ratios the sample size may have to be very large indeed.

One of the most important applications of these results is in calculating confidence intervals. The्ष general method is based on the idea that the statistic of interest (such as the difference between two means of proportions) would have a normal distribution in repeated samples. ${ }^{3}$

1 Atman DG Practica 1991:132-45.

2 Gardner MJ, Altrnan DG. Calculating confidence intervals for proportions and their differences. In: Gardner MJ, Altman DG, eds. Statistics with confidence. London: British Medical Journal, 1989:28-33

3 Gardner MJ, Altman DG, eds. Statistics with confidence. London: British Medical Journal, 1989:17. 